\begin{tabular}{ccc}
\hline & International Journal of Engineering \& Technology, $7(2.17)(2018) 13-16$ \\
SPC & International Journal of Engineering \& Technology \\
Website: $w w w . s c i e n c e p u b c o . c o m / i n d e x . p h p / I J E T$ & Research Paper \\
\hline
\end{tabular}

\title{
Topology Optimization of Dual Octagonal Array antenna for low frequency applications
}

\author{
P. Venu Madhav ${ }^{*}$, Dr. M SivaGanga Prasad ${ }^{2}$ \\ ${ }^{1}$ Department of Electronics \& Communication Engineering, Research Scholar, KL University, Guntur \\ ${ }^{2}$ Department of Electronics \& Communication Engineering, KL University, Guntur \\ *Email:Venu7485@gmail.com
}

\begin{abstract}
Objectives: Efficient antenna design for use in communication systems is altering the face of the antenna modeling. The ever-increasing demand for portable and efficient antennas is making researchers to develop innovative models [1] using advanced antenna modeling tools that comply with industrial needs and standards. Methods/Statistical Analysis: Antennas with lower operating frequencies have the major constraints on its size, efficiency and gain. Study on matching techniques, feeding techniques was also to be considered.

Findings: Micro strip patch antennas offers considerable amount of radiation, low cost when fabricated on FR4, light weight and are conformable to suite any application. This paper projects the design, simulation and testing of a dual octagonal patch, the topology algorithm [3] is used to optimize the size and shape of the patch where octagons are spaced in the form of an array to address optimization on size and fits into wireless applications. Application/Improvements: The proposed model is tested in the standard antenna test bench comprising of microwave integrated circuit analyzer receiver MIC10kit and found to operate at a resonant frequency of $1.8 \mathrm{GHz}$ with good radiation characteristics.
\end{abstract}

Keywords: Micro Strip, Radiation Pattern, Polarization, VSWR, Octagonal Structure, Square Patch, Gain,Bandwidth.

\section{Introduction}

The increase interest in microwave integrated circuits(MIC's) the design of antennas for applications, require a radiating element which offers high directivity, unflattering radiation. The patch and the ground plane are separated by a dielectric substrate in a microstrip. The octagonal shape is used as radiator and this simplifies the analysis and performance prediction. The patch is photo etched on the dielectric substrate(FR4). The relative permittivity of the substrate is an important parameter to consider.

Microstrip patch antennas radiate primarily because of the fringing fields between the patch edge and the ground lane. For good antenna performance, a thick dielectric substrate having a low dielectric constant is desirable since this provides better efficiency, larger bandwidth and the better radiation, but increases the antenna size. To design a compact microstrip patch antenna, higher dielectric constants must be used which are less efficient and result in narrower bandwidth.

Microstrip radiation antenna is a referred choice by many of the researchers because of it offers low profile, high directivity, broadband and compact. The quality factor of the antenna using microstrip is given as

$$
\mathrm{Q}=\left(\omega / 2 \prod\right) \sqrt{\left(\mathrm{dR}_{\mathrm{A}} / \mathrm{d} \omega\right) 2+(\mathrm{dXA} / \mathrm{d} \omega+|\mathrm{XA}| / \omega)}
$$

where $\mathrm{R}_{\mathrm{A}}$ and $\mathrm{X}_{\mathrm{A}}$ are antenna radiation resistance and reactance, respectively. Antenna performances considered are resonance frequency, $\mathrm{Q}$ as a parameter to indicate bandwidth, and resonant radiation resistance. In discussion, the effective volume of spherical radius $\mathrm{r}$ is used as a parameter that relates with $\mathrm{Q}$ and discussed by using normalized $\mathrm{r}$ with the effective height $\mathrm{h}$; that is, $\mathrm{r} / \mathrm{h}$. Several array processing techniques are presented in [11]-[15].

\section{Feeding Mechanism:}

The most effective feed mechanism is a slot line feed. The transition is designed to couple signals to the slot from the transmitter or receiver circuitry. This technique is also easy to fabricate. The feeding technique can be divided into two categories. 1. Directly coupled and 2. Electromagnetically coupled transitions. In a direct coupled transition, a wire or a solder connection act as the direct current path and provides the electrical contact. In electromagnetically coupled transition the coupling of the signal is through electromagnetic fields. In this model, a direct coupled microstrip line feed transition technique is used. The conducting strip is smaller in width as compared to the patch and this kind of feed arrangement has the advantage that the feed can be etched on the same substrate to provide a planar structure.

However, as the thickness of the dielectric substrate being used, increases, surface waves and spurious feed radiation also increases, which hampers the bandwidth of the antenna. The feed radiation also leads to undesired cross polarized radiation. This method is advantageous due to its simple planar structure.

\section{Octagonal Simulation}

The figure 1 below is a 3D view of octagonal structure chosen patch rather than the other shapes. The reason behind it is the earlier research is done on the rectangular, square, circular and hexagon patch microstrip patch antennas. But, the current research is progressing in the dimensional modelling of antenna considering factors that influence the size reduction, shape and other parameters. When 
compared to the rectangular patch, octagonal patch occupies equal or lesser area. As the number of edges is more identification of feed point addressing is made easy which can enhance the radiation pattern and it is easy to operate.

As the name, dual octagonal coplanar structure for wireless applications indicates that we used an antenna array. where antenna array is a set of 2 or more antennas. The signals from the radiator are processed to achieve enhanced performance over that of a single antenna.

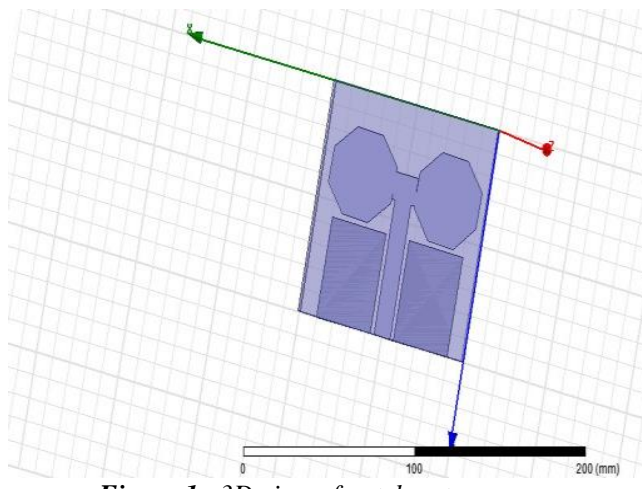

Figure $13 D$ view of patch antenna

These radiators are used to enhance the overall gain and to cancel out nosiness from a set of directions. They can also be used to determine the course of arrival of incoming signals and to maximize the signal to noise ratio.

In the figure 2 above first iteration, an octagonal patch microstrip antenna per our design considerations using high frequency structure simulator (HFSS) software, the antenna resonated at a frequency of $2.6 \mathrm{GHz}$. Later, it was iterated for and the patch is optimized which resonates at a frequency of 1.8 GHz.The designed model is placed in a radiation box which is used for testing an antenna model. It simulates a reflection less free space and allows all weather antenna measurements in a controlled laboratory environment. The isolation can be further improved by shielding.

The topology algorithm [4] has generally been used to optimize size/shape of objective and it provides a possibility of volumetric design. The optimization scheme will bring the best topological and geometrical configuration [5] from its volumetric variation, along with consideration of physical and geometrical dimensions as well as material composite.

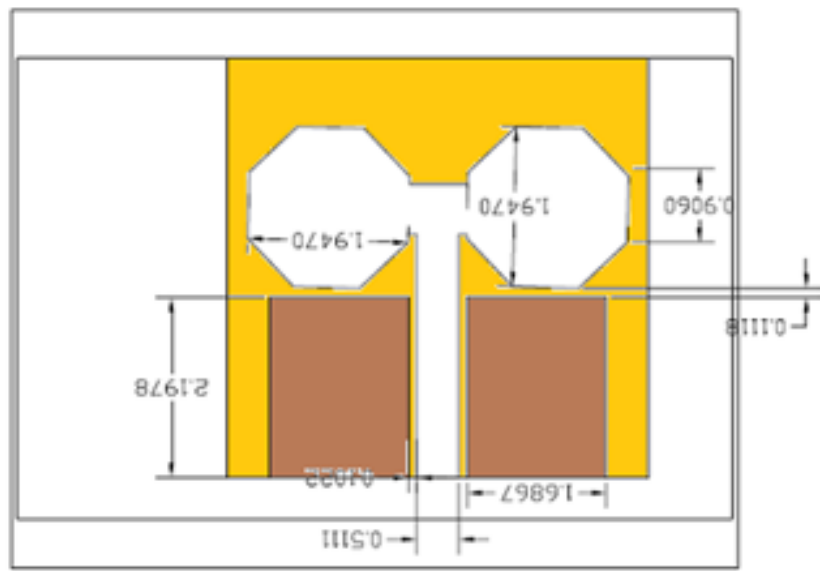

Figure 2 Scale values of the proposed model

It performs iteration of evaluation or each the optimum (maximum) value initially set to obtain the objective shape/configuration. It was demonstrated that the volumetric material design led to significant improvement of bandwidth [6] in a simple patch antenna because of the application of the topology optimization method.

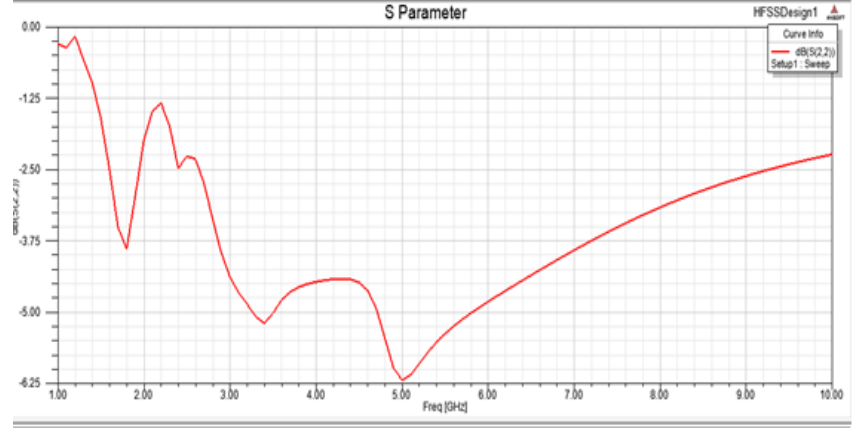

Figure 3 S-Parameter measurement in HFSS
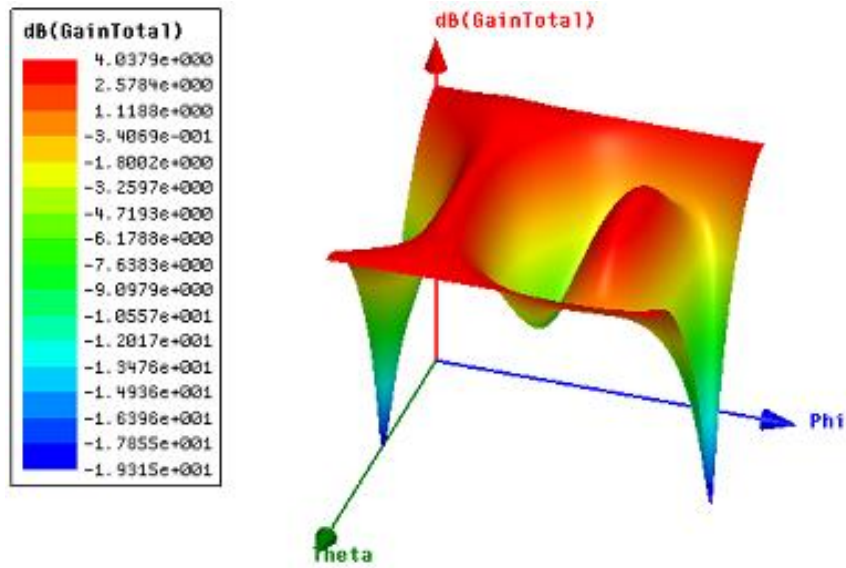

Figure 4 Total Gain in 3-D View

In the design procedure, the feeding technique we opted is coaxial probe feeding. The main advantage of this type of feeding scheme [7] is that the feed can be placed at any desired location inside the patch to match with its input impedance.

$$
w=\frac{c}{2 f_{r} \sqrt{\left(\varepsilon_{r}+1\right) / 2}}=30 \mathrm{~mm}
$$

The bottom of the substrate is covered entirely by a ground plane and offers good fringing field between the radiator and ground. At this point much focus is not given to square patch as ground.

\section{Near Field:}

The field, which is nearer to the antenna, has an inductive effect and hence known as inductive field or near field[8].

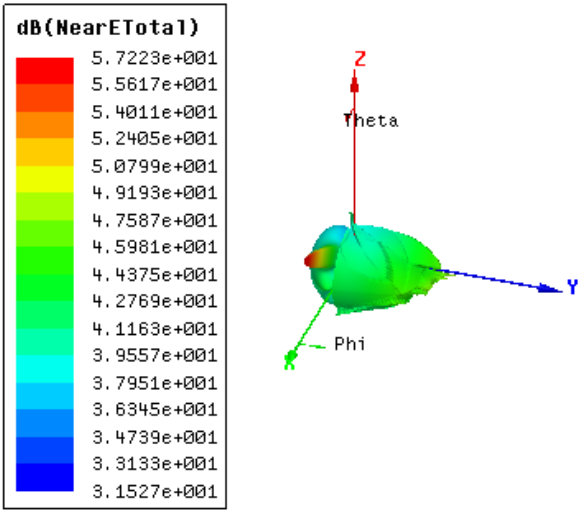

Figure 5 Near Field Gain

\section{Far field:}

The field, which is far from the antenna, is called as radiation field, as the radiation effect is high in this area. Many of the antenna 
parameters along with the antenna directivity and the radiation pattern of the antenna are considered in this region only.

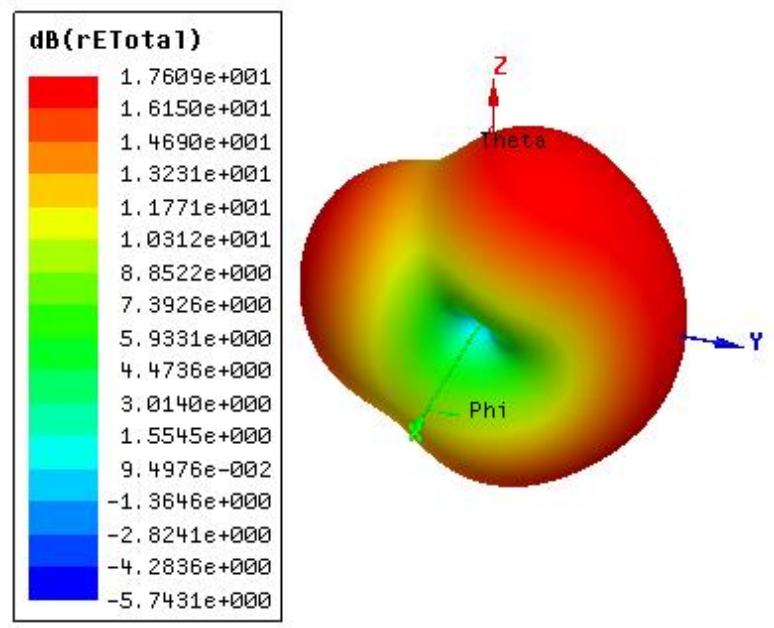

Figure 6 3-D Polar Plot of the proposed model

\section{Antenna measurement:}

Antenna physiognomies are measured using microwave antenna lab. It is very easy to obtain antenna input characteristics using antenna trainer kit. The radiation characteristics of antenna can be measured using MIC10 Microstrip trainer kit consisting of MIC10 microwave integrated circuit analyzer receiver for which the microstrip patch antenna that was fabricated fixed as receiver.

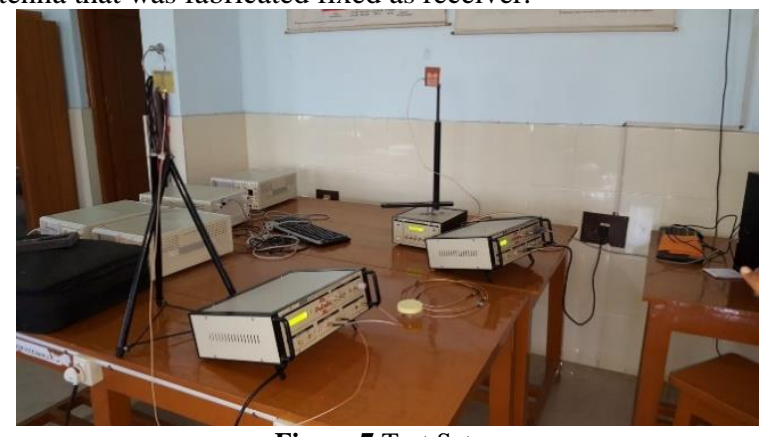

Figure 7 Test Setup

The experimental setup is shown in figure. The distance between transmitting and receiving antennas is approximately 2 meters. Here we used the dipole antenna and microstrip patch antenna. When a standard antenna (dipole antenna) is used as a transmitter and test antenna (microstrip patch antenna) as a receiver, radiation pattern and corresponding gain[9] values are noted. First the dipole must be in horizontal facing vertical receiving antenna to get the copolar of the receiver antenna.

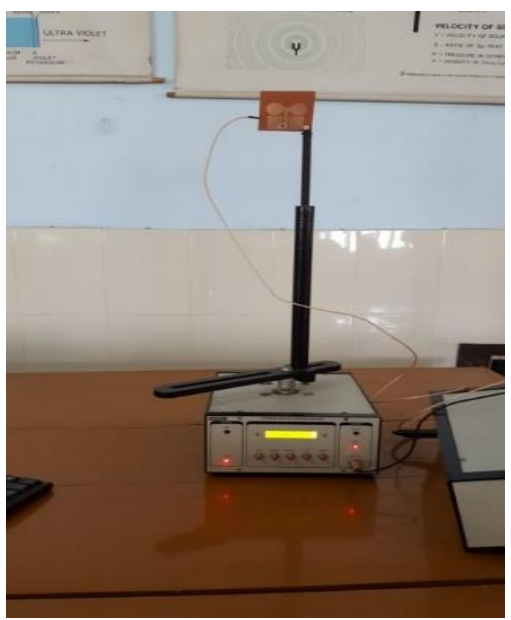

Figure 8 Proposed model as Receiver
A comparison method is adopted for antenna gain measurements. Here, we need one standard antenna. First the dipole antenna is placed on the stepper motor, aligned with the transmitter (Proposed microstrip patch). Next, the standard antenna at receiver is replaced with the test antenna without disturbing the rest of the setup. The test antenna is aligned at the direction of its peak by controlling the stepper motor. Radiation pattern, gain and bandwidth of the test antenna [10] are measured using this setup.

Dipole antenna is connected on tripod to microwave integrated circuit analyzer source MIC10 kit and microstrip patch antenna is connected to stepper motor through microwave integrated circuit analyzer receiver MIC10 kit.

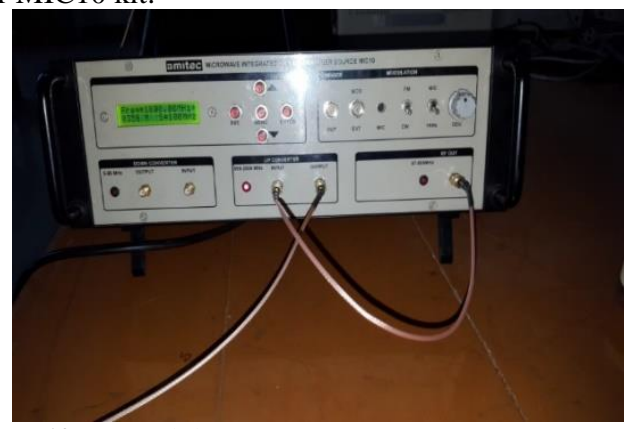

Figure 10. Transmitting Frequency Setup upto $2 \mathrm{GHz}$

\section{Results}

Design and experimental tests were used in order to evaluate the performance of antenna. Experimental results are compared with simulation performance estimates to verify that the design performs as intended. Fabricated and optimized hybrid fractal antenna operating at frequency band of $1.8 \mathrm{GHz}$. The fabricated and the tested antenna which resonates at a frequency of $1.8 \mathrm{GHz}$ have the maximum gain of $40.5 \mathrm{~dB}$. The resultant beam width is $51 \mathrm{~dB}$ and the half power beam width is $25.5 \mathrm{~dB}$. The radiation pattern of the octagonal shape microstrip patch antenna at $1.8 \mathrm{GHz}$ operational frequency:

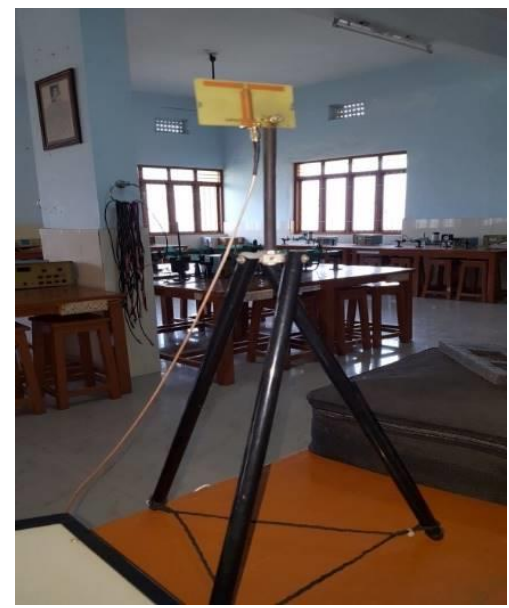

Figure 9 Dipole as transmitter

The fabricated octagonal shape microstrip patch antenna parameters are summarized.

Table 1 Performance parameters of the proposed model

\begin{tabular}{|c|c|}
\hline Performance parameter & Value \\
\hline Operating frequency & $1.8 \mathrm{GHz}$ \\
\hline Max. Gain & $40.5 \mathrm{~dB}$ \\
\hline Dielectric type & FR4 \\
\hline Dielectric constant & 4.36 \\
\hline
\end{tabular}




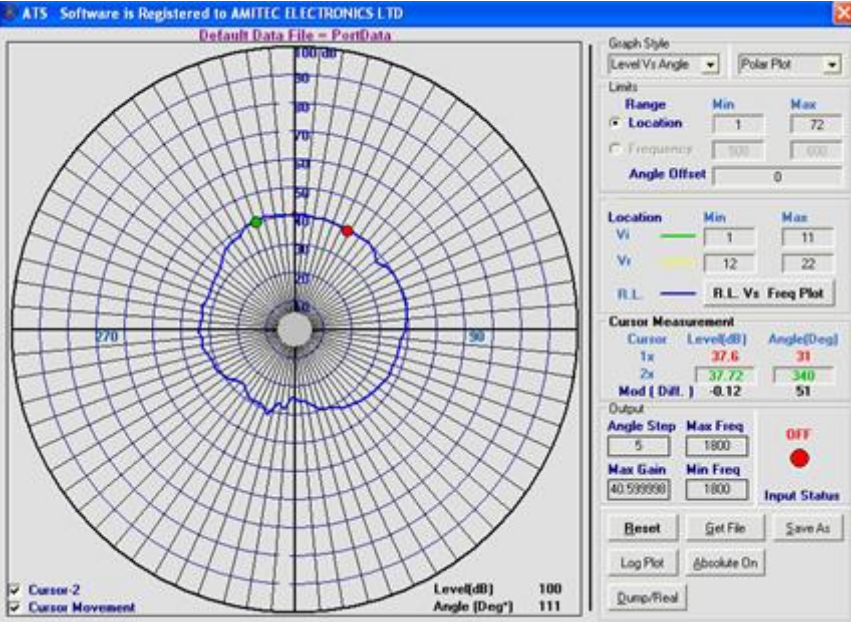

Figure 11. Polar Plot of the proposed model

Level vs. Angle log plot of the octagonal microstrip patch antenna in Cartesian form:

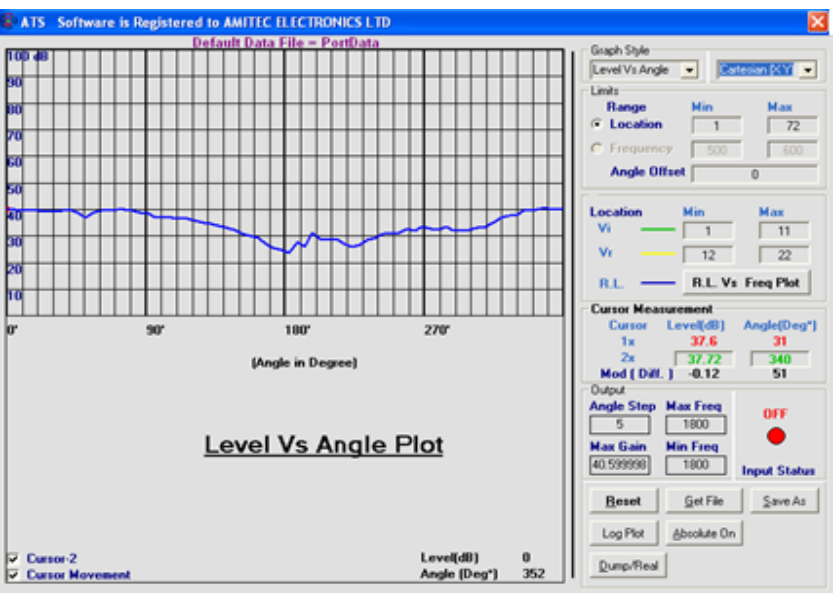

Figure 12 Level Vs angle Plot

\section{Conclusions}

In this work a dual octagonal coplanar structure microstrip patch antenna has been proposed and fabricated for wireless application with an optimization on the patch size with improved gain, bandwidth and radiation pattern. The radiation characteristics of the proposed octagonal microstrip patch antenna have been obtained using antenna software provided by AMITEC, related microstrip workbench compared with those of conventional microstrip patch dipole antenna.

From design and measurement results, radiation pattern of the microstrip antenna was good enough because of the fabrication material and substrate thickness. Further, the gain and radiation pattern can be enhanced by choosing other substrate materials. The proposed antenna has a maximum gain of $40.5 \mathrm{~dB}$, beam width of 51 decibels and half power beam width of $25.5 \mathrm{~dB}$. Therefore, the proposed and designed antenna is feasible for use a low profile and low cost dual band antenna for supporting various wireless communication services.

\section{References}

[1] C. Delabie, M. Villegas, and O. Picon, "Creation of New Shapes for Resonant Microstrip Structures By Means of Genetic Algorithms", Electronics Letters, vol. 33, August 1997, pp. 1509-1510.

[2] P.Venu Madhav, Dr. M Sivaganga Prasad, "Investigation on Octagonal shape complimented Microstrip antenna for low frequency operations using CSA-Algorithm", International Journal of Scientific \& Engineering Research Volume 8, Issue 8, August2017 pp790-795, ISSN 2229-5518
[3] Prem Narayan Choubey, Wei Hong, Zhang-Cheng Hao, Peng Chen, Tuan-Viet Duong, and Jiang Mei, "A Wideband DualMode SIW Cavity-Backed Triangular-Complimentary-SplitRing-Slot (TCSRS) Antenna," IEEE TRANSACTIONS ON ANTENNAS AND PROPAGATION, VOL. 64, NO. 6, JUNE 2016.

[4] G. Kiziltas and D. Psychoudakis, J. L. Volakis, and N. Kikuchi, "Topology design optimization of dielectric substrates for bandwidth improvement of a patch antenna", IEEE Transactions on Antennas and Propagation, vol. 51, October 2003, pp. 2732-2743.

[5] A. M. Montaser, K. R. Mahmoud, Adel B. Abdel-Rahman, H. A Elmikati Senior Member IEEE, "CIRCULAR, HEXAGONAL AND OCTAGONAL ARRAY GEOMETRIES FOR SMART ANTENNA SYSTEMS USING HYBRID CFO-HC ALGORITHM," Journal of Engineering Sciences, Assiut University, Vol. 40 No 6 pp.1715-1732 - November 2012.

[6] T. Nomura, K. Sato, S. Nishiwaki, and M. Yoshimura, Topology Optimization of Multiband Dielectric Resonator Antennas Using Finite-Difference Time-Domain Method, IEEE International Workshop on Antenna Technology, March 2007, pp. 147-150.

[7] P Venu Madhav, Dr. M. Sivaganga Prasad, "A New Geometrical Design of Octagonal Fractal Microstrip Patch Suitable For mobile \& Wi-Max Applications", IJECT Vol. 5, Issue spl - 3, Jan - March 2014 ISSN : 2230-7109 (Online) | ISSN : 2230-9543.

[8] G. Kiziltas, and J. L. Volakis, Shape and Material Optimization for Bandwidth Improvement of Printed Antennas on High Contrast Substrates, IEEE International Symposium on Electromagnetic Compatibility, 2003. EMC'03, vol. 2, 11-16 May 2003, pp. 1081-1084.

[9] E. Saenz et al., Coupling Reduction Between Dipole Antenna Elements by Using a Planar Meta-Surface, IEEE Transactions on Antennas and Propagation, vol. 57, 2009, no. 2, pp. 383-392.

[10] P VenuMadhav, Dr. M. Sivaganga Prasad, "Interleaved Metallic Octagonal Structures for Low Frequency Applications", International Journal of Control Theory and Applications, ISSN: 0974 5572, International Science Press, Volume 10 • No. 352017

[11] M.L.M. Lakshmi, K.Rajkamal, S.V.A.V.Prasad, Md.Zia Ur Rahman, "Amplitude Only Linear Array Synthesis With Desired Nulls Using Evolutionary Computing Technique", The Applied of Computational Electromagnetics Society Journal, vol.31, no.11, pp. 1357-1361, November, 2016.

[12] Y. Murali Krishna, Md. Zia Ur Rahman and B.V. Rama Mohana Rao, "Beam Steering in Smart Antennas using an Efficient Adaptive Signal Processing Algorithm," International Journal of Research and Reviews in Signal Acquisition and Processing, Vol. 1(3), Sep. 2011.

[13] K. Sarath Kumar, Md. Zia Ur Rahman, "A New Computation Method for Pointing Accuracy of Cassegrain Antenna in Satellite Communication", Journal of Theoretical and Applied Information Technology,Vol.95, no.13, 2017, pp.3062-3074.

[14] Md. Zia Ur Rahmna, K. Murali Krishna, "Efficient Adaptive Beamforming Algorithms for Smart Antennas", International Journal of Control Theory and Applications,Vol.10, no.35, 2017, pp.173-181.

[15] Md. Zia Ur Rahman, et al., "A Low Complex adaptive algorithm for Antenna beam steering", IEEE 2011 International Conference on Signal Processing, Communications, Computing and Networking Technology (ICSCCN 2011), ISBN: 978-1-61284-6538, pp.317-321, 2011. 